\title{
Avaliação dos Métodos Empregados no Programa Nacional de Combate ao Câncer do Colo Uterino do Ministério da Saúde
}

\author{
Evaluation of the Methods Employed by the National Program of Uterine Cervical \\ Cancer Control of the Brazilian Health Ministry \\ Alfredo Roberto Neto, Julisa Chamorro Lascasas Ribalta \\ José Focchi, Edmund Chada Baracat
}

\begin{abstract}
RESUMO
Objetivo: avaliar uma amostra populacional incluida no rastreamento proposto pelo Programa Nacional de Combate ao Câncer do Colo do Utero (PNCC), quanto aos seguintes aspectos: prevalência de resultados citológicos insatisfatórios; prevalência citológica de atipias escamosas e glandulares de significado indeterminado (ASCUS e AGUS) e lesões intraepiteliais de baixo grau (LBG) e lesões intra-epiteliais de alto grau (LAG), comparando seus resultados com resultados anatomopatológicos de biópsias colposcopicamente dirigidas.

Métodos: pela imprensa escrita, falada e televisionada, foram convocadas mulheres, com idade entre 35 e 49 anos, para realização de exames citopatológicos preventivos, a serem colhidos nas unidades da rede de saúde pública ou instituições credenciadas pelo SUS. As lâminas foram analisadas por laboratórios credenciados pelo PNCC, e as pacientes com alterações celulares da amostra populacional do Municipio de Naviraí/MS foram submetidas a exame colposcópico e biópsia dirigida.

Resultados: a prevalência de alterações citológicas do tipo ASCUS, AGUS e lesões escamosas intra-epiteliais foi de 3,3\%, indice próximo do previsto pelo PNCC (4\%). O percentual de espécimes insatisfatórios para avaliação foi elevado (12,5\%); das pacientes com resultados citológicos de ASCUS, AGUS ou LBG, 27,3\% apresentavam neoplasia intra-epitelial de alto grau ao estudo anatomopatológico. Por outro lado, nas pacientes com citologia compativel com LAG a biópsia dirigida revelou, em 12,5\% dos casos, neoplasia intra-epitelial de baixo grau.

Conclusões: a escolha da citologia oncótica como método único de rastreamento no PNCC permitiu elevados indices de falso-negativos (27,3\%) e de falso-positivos (12,5\%). No rastreamento de neoplasias cervicais, a colposcopia mostrou-se procedimento importante $e$ indispensável para nortear as condutas terapêuticas a serem adotadas.
\end{abstract}

PALAVRAS-CHAVE: Colo: câncer. Lesões pré-neoplásicas. Rastreamento para câncer. Colpocitologia.

\section{Introdução}

Segundo dados da Organização Mundial de Saúde ${ }^{1}$, a partir de 2020 estima-se no mundo o diagnóstico de 15 milhões de novos casos de câncer ao ano. Mantidas as condições socioculturais atu-

Departamento de Ginecologia, Setor de Patologia do Trato Genital Inferior e Colposcopia, Universidade Federal de São Paulo - Escola Paulista de Medicina, UNIFESP-EPM Correspondência:

Alfredo Roberto Netto

Av. Dourados, 569

79950-000 - Naviraí - MS

e-mail: roberti@uol.com.br ais, cerca de $70 \%$ desses tumores ocorrerão em países dos quais apenas $5 \%$ possuem recursos para controle da doença ${ }^{1}$. Aplicando-se o conhecimento científico que se possui, poder-se-ia reduzir cerca de um quarto a incidência de todos os cânceres e curar a terça parte deles, com a tecnologia atual. Com essas medidas, reduzir-se-ia a incidência da doença à metade nos próximos $25 \operatorname{anos}^{1}$.

O câncer do colo do útero representa a segunda causa de morte por câncer de mulheres no Brasil, superado apenas pela neoplasia de mama ${ }^{2}$. Constitui um problema de saúde pública e é doença passivel de ser prevenida, estando diretamente vinculada ao grau de subdesenvolvimento do país ${ }^{2}$. 
Em 1996, o Ministério da Saúde investiu $\mathrm{R} \$ 854.000,00$ (oitocentos e cinqüenta e quatro mil reais) na organização e estrutura de seu Banco de Dados, além de contratar a Consultoria da "Cancer Care International" (CCI), braço internacional da Fundação Ontário para o Tratamento e Pesquisa do Câncer (Canadá). Essas medidas visavam a prestação de serviços e assistência técnica na elaboração de um programa nacional de combate ao câncer de colo uterino ${ }^{2}$.

No ano de 1998, motivado pelos dados estatísticos, retrospectivos e prospectivos, embasado na orientação da CCI, o Ministério da Saúde instituiu o Programa Nacional de Combate ao Câncer do Colo Uterino - $\mathrm{PNCC}^{2}$.

Elegendo o exame citológico de Papanicolaou como método único de rastreamento, e cirurgia de alta freqüência (CAF), quando possivel, para tratamento das lesões intra-epiteliais pelo método "Ver e Tratar", estabeleceram-se também as metas e estimativas para o programa. Definiram-se a estratégia organizacional, as intervenções, os critérios para diagnóstico e as condutas clínicas diante dos resultados dos exames citológicos.

Dentro dos critérios para diagnósticos estabelecidos, o PNCC definiu como lesões intraepiteliais de baixo grau (LBG) ${ }^{2}$ as alterações citológicas induzidas pelo papilomavírus humano (HPV), atipias de células escamosas de caráter desconhecido (ASCUS), atipias de células glandulares de significado indeterminado (AGUS) e neoplasia intra-epitelial grau I (NIC I) ${ }^{2}$; as alterações NIC II e III (neoplasias intra-epiteliais de graus II e III) foram classificadas como lesões intra-epiteliais de alto grau (LAG).

No que tange às condutas clínicas, orientou que, se o resultado do exame citopatológico fosse compativel com ASCUS, AGUS, HPV ou NIC I, dever-se-ia tratar os processos inflamatórios com a terapêutica definida pelo PNCC e repetir o exame após seis meses. Em persistindo as alterações no exame citológico de repetição, dever-se-ia, então, encaminhar a paciente para exame colposcópico. Em caso contrário, repetir novo exame citológico, somente após três anos.

Se o resultado do exame citopatológico apresentasse NIC II, NIC III, carcinoma escamoso invasivo, adenocarcinoma in situ, adenocarcinoma invasivo ou outras neoplasias malignas, dever-seia encaminhar a paciente para exame colposcópico e, na presença de lesão delimitada e junção escamocolunar visivel, aplicar o método "Ver e Tratar" pela CAF, com exérese total da lesão.

O seguimento dependeria do exame anatomopatológico. Se o espécime retirado fosse insatisfatório, dever-se-ia repetir a colposcopia e a citologia após dois meses. Por outro lado, se após a retirada total da lesão, o resultado anatomopato- lógico desvelasse NIC III, dever-se-ia repetir o exame citológico após seis meses. Se o espécime retirado representasse retirada parcial da lesão com laudo anatomopatológico até NIC III, dever-se-ia realizar a conização a bisturi; quando o espécime retirado apresentasse resultado de carcinoma escamoso invasivo, adenocarcinoma in situ, adenocarcinoma invasivo ou outras neoplasias malignas, dever-se-ia encaminhar a paciente para unidade terciária de tratamento.

Analisando 125 pacientes, Swinker et al. ${ }^{3}$ observaram grandes divergências entre os esfregaços citológicos e os resultados das biópsias dirigidas; achados citológicos de alto grau têm valor preditivo para alterações histológicas de alto grau, porém, os achados citológicos de baixo grau necessariamente não indicam doenças de baixo grau. Consideram que apenas o uso de esfregaço citológico para pesquisar anormalidades cervicais ou NICs de alto grau resulta em estimativa grosseira na prevalência e gravidade da doença, deixando de tratar ou postergando tratamento em $21 \%$ das pacientes. Recomendam que todas as citologias que demonstram displasias ou inflamações com atipias devam ser submetidas à colposcopia e biópsia dirigida.

Discordando, portanto, da postura do PNCC por entender que todas as alterações citológicas deveriam ser submetidas à avaliação colposcópica com biópsia dirigida, quando fosse julgado necessário, e com o objetivo de comprovar esta assertiva, os autores se propuseram a submeter um grupo de mulheres participantes do PNCC à triade diagnóstica: citologia - colposcopia - histologia, e comparar seus resultados com os do programa.

Objetiva-se no presente trabalho avaliar o rastreamento proposto pelo PNCC do Ministério da Saúde do Brasil quanto aos aspectos da prevalência de resultados citológicos insatisfatórios, da prevalência citológica de ASCUS, AGUS e lesões escamosas intra-epiteliais cervicais e do diagnóstico histopatológico de casos com resultados citológicos de ASCUS, AGUS, LBG e LAG.

\section{Pacientes e Métodos}

Para a efetivação do PNCC, a população feminina brasileira foi convocada pela imprensa escrita, falada e televisionada segundo o protocolo estabelecido pelo Ministério da Saúde, para a coleta do esfregaço de Papanicolaou. Estabeleceu-se, como prioridade, o atendimento de mulheres com idade entre 35 e 49 anos e aquelas que nunca haviam sido submetidas a esse exame, ainda que as pertencentes a outras faixas etárias pudessem ser atendidas. 
A coleta dos esfregaços citológicos foi realizada por integrantes das unidades de saúde dos Municípios, assim como por estabelecimentos privados vinculados à rede de saúde pública, via Sistema Único de Saúde - SUS.

As metas e parâmetros foram transmitidos aos coordenadores estaduais, que se incumbiram de repassá-los às suas áreas de atuação e promover os treinamentos necessários para sua execução.

O Ministério da Saúde adquiriu o material para a coleta da citologia, segundo estimativa pré-estabelecida, disposto em conjuntos compostos de espéculo descartável, um par de luvas descartáveis, uma escova cervical, uma lâmina de vidro, uma espátula de Ayre, um frasco de fixador de esfregaço e uma pequena caixa para acondicionamento das lâminas após a coleta. Ficou sob a responsabilidade das Secretarias de Saúde dos Estados a distribuição dos conjuntos para suas unidades.

A confecção de esfregaço citológico, a ser analisado, resultou de dupla coleta, ou seja, material endo e ectocervical. Segundo a coordenação da campanha, como o colo uterino repousa em contato com o fundo vaginal e suas paredes, estaria ele imerso no conteúdo vaginal representado pelos elementos celulares de todas as origens, sendo desnecessário a coleta do fórnice posterior da vagina.

A lâmina, após fixada, era remetida para laboratórios credenciados pelo PNCC para a devida leitura citológica, via Secretaria Municipal de Saúde. Os resultados retornaram aos municipios e foram tratados segundo os parâmetros e condutas clínicas definidas pelo PNCC.

As pacientes portadoras de atipias celulares de significado indeterminado (ASCUS e AGUS) e as neoplasias intra-epiteliais grau I (NIC I) foram tratadas, conforme protocolo clínico e terapêutico estabelecido pelo PNCC, por profissional médico definido pela Secretaria Municipal de Saúde de cada município. Da mesma forma, o tratamento daquelas com exames compativeis com LAG, por avaliação colposcópica seguida de cirurgia de alta freqüência - $\mathrm{CAF}$, ou encaminhamento das mesmas para tratamento terciário na eventual impossibilidade de se visualizar a lesão, foi feito por profissional previamente designado.

No município de Naviraí/MS, um dos autores foi o responsável pelo tratamento das LBG e das LAG resultantes da avaliação citológica de 2.278 mulheres durante o PNCC.

Sem qualquer prejuízo para as pacientes, previamente informadas, e de comum acordo com a Secretaria Municipal de Saúde, o autor dispôsse a seguir conduta diferente, submetendo todas as pacientes com atipias de significado indeterminado, LBG e LAG à colposcopia e biópsia dirigida precedendo a opção terapêutica pelo CAF.

O protocolo desta versão foi aprovado pela Comissão de Ética em Pesquisa da Universidade Federal de São Paulo - Escola Paulista de Medicina-UNIFESP-EPM.

$O$ que se segue representa o resultado do estudo das alterações citológicas ASCUS, AGUS, LBG e LAG encontradas apenas no município de Naviraí/MS. Os esfregaços citológicos foram analisados por laboratórios credenciados pelo PNCC, fora do Município de Naviraí, segundo os critérios da Secretaria Estadual de Saúde.

Foi utilizado para a avaliação colposcópica aparelho binocular, marca DFV - DF Vasconcelos, modelo 18461, com cinco aumentos, e de estativa fixa à mesa ginecológica. A notação das imagens encontradas fez-se segundo a nomenclatura de aspectos colposcópicos estabelecida durante o VI Congresso Mundial de Patologia Cervical e Colposcopia sediado em Roma em 1990.

Os espécimes de biópsias colhidos com pinça de Gaylor-Medina, assim como aqueles decorrentes das cirurgias de alta freqüência, foram acondicionados em frascos com solução de formol a $10 \%$. Os exames anatomopatológicos foram realizados no Laboratório Central do Estado do Mato Grosso do Sul, na cidade de Campo Grande.

\section{Resultados}

Das 2.278 mulheres examinadas pelo esfregaço cervical, segundo a técnica de Papanicolaou, 1.918 apresentaram resultados dentro dos limites da normalidade; 57 revelaram ASCUS, AGUS ou LBG (HPV e NIC I); 16 LAG (NIC II e III) e 2 cânceres invasores. Do total, 285 exames foram considerados insatisfatórios (Tabela 1).

O protocolo original de critérios de tratamento do PNCC propunha que as alterações celulares de significado indeterminado, as infecções por HPV e as NICs I fossem tratadas igualmente, ou seja, recebesse a terapêutica medicamentosa preestabelecida e/ou conduta expectante, com esfregaço de repetição após seis meses, quando então, em persistindo as alterações, deveriam ser encaminhadas para exame colposcópico. Por essa razão, no presente trabalho, ASCUS, AGUS, HPV e NIC I serão analisadas em conjunto. A faixa etária das mulheres que compunham esse grupo variou de 18 a 48 anos, com média de idade de 29,7 anos. À colposcopia, 24 apresentaram achados colposcópicos normais e as demais, aspectos anormais, como pode ser visto na Tabela 2. 
Tabela 1 - Distribuição dos resultados citológicos de 2.278 mulheres do Município de Naviraí/MS, segundo a presença ou não de atipias celulares - PNCC - 1998.

\begin{tabular}{lcc}
\hline Resultados & $\mathbf{n}$ & $\mathbf{\%}$ \\
\hline Normais & 1.918 & 84,2 \\
Exames insatisfatórios & 285 & 12,5 \\
ASCUS & 25 & 1,1 \\
AGUS & 12 & 0,5 \\
LBG & 20 & 0,9 \\
LAG & 16 & 0,7 \\
Câncer invasor & 2 & 0,1 \\
Total & 2.278 & 100,0 \\
\hline
\end{tabular}

ASCUS: Atipia de célula escamosa de significado indeterminado

AGUS: Atipia de célula glandular de significado indeterminado

LBG: Lesão de baixo grau

LAG: Lesão de alto grau

Tabela 2 - Resultados do exame colposcópico de 57 mulheres com ASCUS, AGUS e LBG à colposcopia.

\begin{tabular}{llr}
\hline Resultados colposcópicos & $\mathbf{n}$ & $\%$ \\
\hline Normais & 24 & 42,1 \\
Anormais & 33 & 57,9 \\
Total & 57 & 100,0 \\
\hline
\end{tabular}

Na Tabela 3 observa-se que, entre as pacientes que apresentavam ASCUS, AGUS e LBG à citologia e que tinham anormalidades colposcópicas, $27(81,8 \%)$ mostraram também alterações anatomopatológicas. Ressalta-se que em nove $(27,3 \%)$ foram encontradas lesões de maior gravidade, NIC II e NIC III.

Tabela 3 - Distribuição de 57 pacientes com resultados citológicos com ASCUS, AGUS e/ou LBG, segundo a avaliação colposcópica.

\begin{tabular}{lrr}
\hline Atipias colposcópicas & $\mathbf{n}$ & \% \\
\hline Normais & 24 & 42,2 \\
Condiloma & 1 & 1,8 \\
EAB & 12 & 21,0 \\
Pontilhado & 7 & 12.3 \\
Mosaico & 4 & 7,0 \\
EAB denso + pontilhado & 5 & 8,7 \\
EAB denso + mosaico & 3 & 5,2 \\
Mosaico + vaso atípico & 1 & 1,8 \\
Total & 57 & 100,0 \\
\hline
\end{tabular}

EAB: Epitélio aceto-branco

ASCUS: Atipia de célula escamosa de significado indeterminado

AGUS: Atipia de célula glandular de significado indeterminado

LBG: Lesão de baixo grau
As dezesseis pacientes portadoras de esfregaços compativeis com LAG foram também submetidas à avaliação colposcópica. Todas apresentaram aspectos colposcópicos anormais (Tabela 4). A distribuição etária dessas mulheres variou de 28 a 51 anos, com média de 36,3 anos.

Tabela 4 - Distribuição de 33 pacientes com resultados citológicos compatíveis com ASCUS, AGUS e/ou LBG, segundo os resultados do exame anatomopatológico.

\begin{tabular}{lrr}
\hline Resultados anatomopatológicos & $\mathbf{n}$ & $\%$ \\
\hline Cervicite crônica & 2 & 6,1 \\
Metaplasia & 4 & 12,1 \\
NBG & 18 & 54,5 \\
NAG & 9 & 27,3 \\
Total & 33 & 100,0 \\
\hline
\end{tabular}

NBG: neoplasia intra-epitelial de baixo grau

NAG: neoplasia intra-epitelial de alto grau

Após a biopsia dirigida, o estudo anatomopatológico desses casos revelou a ocorrência de neoplasia intra-epitelial de alto grau em $87,5 \%$ das vezes (14 pacientes). Em 2 casos (12,5\%) o exame anatomopatológico foi de menor gravidade do que o laudo citopatológico (Tabela 5).

Tabela 5 - Distribuição de 16 pacientes portadoras de citologia oncótica compativel com lesão de alto grau segundo os achados colposcópicos.

\begin{tabular}{lcc}
\hline Achados colposcópicos & $\mathbf{n}$ & $\%$ \\
\hline EAB & 2 & 12,7 \\
Pontilhado & 3 & 18,7 \\
EAB + pontilhado & 5 & 31,2 \\
Mosaico grosseiro & 3 & 18,7 \\
EAB denso + mosaico & 3 & 18,7 \\
Total & 16 & 100,0 \\
\hline
\end{tabular}

EAB: Epitélio aceto-branco

\section{Discussão}

"Em 1998, o Ministério da Saúde do Brasil lançou um programa para a detecção do câncer do colo do útero. Em seis semanas, foram examinadas 3,263 milhões de mulheres. Foram identificadas, nesse universo, 1,2 milhão de mulheres com algum tipo de infecção vaginal e 53,9 mil mulheres com câncer de colo uterino. Do total dos casos positivos para câncer, 49,2 mil estavam no estágio inicial da doença ou com alguma lesão 
precursora e 4,7 mil mulheres encontravam-se em estágio de câncer invasivo. A importância do programa pode ser avaliada pela quantidade de diagnósticos da doença no estágio inicial, já que nessa fase o câncer de colo de útero tem cura em 100\% dos casos “(transcrição literal do Programa Nacional de Combate ao Câncer do Colo do Útero do Ministério da Saúde do Brasil)"2.

Em todo o Brasil, no ano de 1998, segundo estatística do Ministério da Saúde, o Sistema Único de Saúde registrou que apenas $550.000 \mathrm{mu}-$ lheres foram submetidas a coleta da citologia de Papanicolaou por mês. Durante o PNCC elevou-se a marca da coleta de exames citológicos para 3,263 milhões, em apenas 45 dias.

No Município de Naviraí/MS, das 2.278 citologias colhidas, 285 (12,51\%) resultaram insatisfatórias. Esse elevado índice sugere falha na coleta ou insegurança na leitura das lâminas. Utilizando-se essa porcentagem como referência para o número total de mulheres rastreadas em todo o país, estima-se que cerca de $408.233 \mathrm{mu}-$ lheres apresentariam citologias insatisfatórias, ou seja, quase a produtividade mensal de citologias que se realizava antes do programa. Obviamente os indices de citologias insatisfatórias devem variar para mais ou para menos, de acordo com a região sob análise.

Para a adequada execução do projeto devem ser definidos requisitos de experiência em todos os niveis de serviço: os educadores, os responsáveis pela coleta do esfregaço, os responsáveis pela leitura dos esfregaços, os responsáveis pelo tratamento e os responsáveis pelo sistema de informações. Devem-se assegurar os métodos, com supervisão de qualidade; prover os locais com todos os niveis de funcionários, com treinamento, com motivação e com a monitorização da qualidade desse grupo de servidores, além de sua qualificação formal ${ }^{4}$.

As 57 pacientes com alterações citológicas referidas como ASCUS, AGUS, LBG e LAG encontradas na amostragem em estudo correspondem a $3,3 \%$ dos casos, percentual bem próximo àquele esperado pela organização do PNCC, que era de 4,0\%.

Das 57 pacientes portadoras de esfregaços contendo ASCUS, AGUS ou LBG, 24 (42,1\%) não apresentaram alterações colposcópicas. Nas restantes 33 mulheres $(57,9 \%)$ foram detectadas alterações colposcópicas e histológicas. Os resultados anatomopatológicos evidenciaram 6 casos com quadro histológico dentro da normalidade $(18,2 \%), 18$ com neoplasias intra-epiteliais de baixo grau $(54,5 \%)$ e outras 9 pacientes com lesões de alto grau $(27,3 \%)$.

Lachman e Cavallo-Calvanese ${ }^{5}$ registraram $36,2 \%$ de alterações histológicas em seus estudos em portadoras de ASCUS (19,5\% de LBG e 16,7\% de LAG). Ainda estudando ASCUS, Yang e

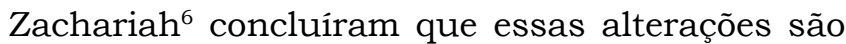
boas indicadoras de NIC. Sugerem que avaliações com biópsia dirigida pelo exame colposcópico são mais eficientes para detectar neoplasias intraepiteliais do que apenas esfregaços de repetição. Do mesmo modo, Zweizig et al. ${ }^{7}$ consideram a presença de AGUS, na citologia cervical, importante indicador de neoplasia ginecológica. Assim, em seu entender, esses casos deveriam ser investigados com colposcopia, biópsia dirigida, curetagem cervical e até, em mulheres mais idosas, biópsia endometrial.

$\mathrm{Na}$ presente pesquisa, as avaliações colposcópicas e anatomopatológicas permitiram detectar que $87,5 \%$ das 16 mulheres com resultados citológicos de lesão de alto grau realmente apresentavam NIC II e NIC III. O uso de cirurgia de alta freqüência, como previsto pelo PNCC, foi, portanto, desnecessário em $12,5 \%$ desses casos cujas biópsias revelaram apenas lesões de baixo grau.

Comparando os resultados da citologia e da biópsia dirigida nas LAG identificadas em produtos de conizações, Heatley e Bury ${ }^{8}$ encontraram 49 e $63 \%$ de concordância entre os resultados, concluindo ser a biópsia o método mais preciso para a estimativa do grau da lesão intra-epitelial.

Foram confirmados no presente estudo dois cânceres invasores detectados pela citologia. Pelo protocolo do PNCC, essas neoplasias seriam imediatamente encaminhadas à CAF. No entanto, realizou-se o estadiamento do tumor e o devido encaminhamento para o tratamento terciário, após a biópsia dirigida.

$\mathrm{Na}$ avaliação de Collaço et al. ${ }^{9}$, no programa de rastreamento do Estado do Paraná, projeto de iniciativa do próprio Estado, onde se utilizou a citologia de Papanicolaou e a CAF no método "Ver e Tratar", somente 53,3\% das pacientes estudadas apresentaram correlação exata entre os diagnósticos citológico e histológico, ou seja, 46,7\% dos casos foram discordantes. De nove casos diagnosticados citologicamente como sendo carcinoma invasivo, não foi encontrada a lesão em 2 (22,2\%); em outros $5(55 \%)$ a lesão não era invasora.

Questiona-se o modelo "Ver e Tratar" como método de rastreamento e tratamento no programa brasileiro, uma vez que, mesmo nos países desenvolvidos, os índices de concordância da citologia e histologia, após a ablação, não justificam a substituição da biópsia colposcopicamente dirigida. A cirurgia de alta freqüência deve ser, sim, o tratamento final para as neoplasias intraepiteliais de alto grau, diagnosticadas pela tríade citologia, colposcopia e histologia, quando houver indicação.

Até o presente momento, a organização do PNCC não concluiu a análise total dos dados levantados em 1998, não permitindo um estudo com 
resultados mais precisos, considerando as diferenças regionais culturais e técnicas próprias do Brasil. Certamente os valores por nós encontrados não devem ser os mesmos observados em cada região do território nacional. No entanto, são suficientes para demonstrar que a não realização da colposcopia, da biópsia dirigida e do estudo anatomopatológico permitiu índices expressivos de falso-negativos $(47,3 \%)$ e falso-positivos $(12,5 \%)$ no rastreamento do câncer cervical.

As médias de idade das pacientes com lesões de baixo grau, 29,7 anos, e 36,3 anos para as com lesões de alto grau demonstram que a maior incidência das lesões precursoras ocorre em faixas etárias inferiores à prevista como prioritária pelo PNCC, que era de 35 a 49 anos.

Estudos recentes ${ }^{10}$ sugerem que o rastreamento deve ser mais freqüente em grupo de mulheres jovens, em função do aumento na incidência de lesões de alto grau entre as pacientes com 20 a 34 anos de idade. Na Suíça, foi observado aumento do número de adenocarcinomas do colo nas mulheres com idade de 25 a 39 anos, apesar de haver um programa de rastreamento em efetivo funcionamento há várias décadas ${ }^{11}$.

Nossos resultados alertam para a necessidade da complementação colposcópica e anatomopatológica nos casos com esfregaços citológicos alterados, principalmente em regiões, onde a qualidade dos exames citológicos da rede pública, de forma geral, pode e deve ser questionada.

Dentre outros, Flannelly et al. ${ }^{12}$, em estudo prospectivo randomizado de 793 mulheres, assinalaram que a supervisão exclusivamente citológica não é estratégia eficiente para acompanhamento de mulheres com esfregaços cervicais com alterações leves, e qualquer grau de discariose deve ser encaminhado para colposcopia.

De acordo com modelo clássico de rastreamento de neoplasia cervical, vigente nos Estados Unidos da América, as mulheres devem se submeter ao exame citológico desde o inicio da atividade sexual, repetindo-o anualmente. Já no Canadá recomenda-se a repetição a cada três anos e na Finlândia, a cada cinco anos por todo seu tempo de vida ${ }^{13}$. Esses parâmetros se baseiam na experiência de cada país, segundo suas realidades, após décadas de estudos e aprimoramento.

A organização do PNCC, no Brasil, demandou 120 dias, entre sua organização e implantação. Efetivou-se como uma campanha. O acompanhamento das mulheres cujos esfregaços apresentaram alterações expressou-se de forma aleatória e irregular. Em 1997, Herbert ${ }^{14}$ já alertava que um programa de rastreamento necessita de tempo para apresentar seus efeitos e que os seus participantes devem receber treinamento, educação continuada e controle de qualidade para alcançar seus objetivos.

Tornar eficiente a estrutura de nosso Sistema de Saúde, efetivar um planejamento prospectivo, com investimento na educação e na capacitação profissional, além da implantação de infra-estrutura humana e material é o caminho para programas dessa natureza, até que novas técnicas, menos onerosas e capazes de alcançar grandes populações, se efetivem. Some-se a isso um acompanhamento continuado. Com essas ações, certamente a qualidade da coleta de esfregaços cervicais e seus resultados apresentarão indices bem menores de falhas e tornar-seão mais confiáveis.

Tais procedimentos também fundamentam as conclusões do "National Workshop on Screening for Cancer of the Cervix"15, conferência de especialistas que analisou o programa nacional canadense de rastreamento do câncer do colo do útero e a base do programa de rastreamento cervical mexicano implantado em $1998^{16}$.

O PNCC foi a primeira iniciativa expressiva do Ministério da Saúde do Brasil, no combate ao câncer cervical invasivo. Intitulado de Programa, pela forma como se expressou, foi apenas uma campanha. Sob essa ótica tem seus méritos e seus resultados deverão diminuir os índices de incidência e de mortalidade pelo câncer cervical, ainda que de maneira pouco expressiva e temporário, uma vez que ainda não se efetivou um planejamento devidamente estruturado e adequado à realidade brasileira.

Durante muitas décadas, a citologia, a colposcopia e a histologia constituem o padrão no rastreamento do câncer cervical nos países desenvolvidos. Acredita-se que esses exames sejam perfeitamente adequados à realidade brasileira, tanto pela sua sensibilidade e especificidade, quanto pelo seu custo. Investir na capacitação profissional, em todos os níveis, e em uma estrutura básica permitirá que a citologia ocupe o seu merecido lugar no PNCC. A aplicação da colposcopia e da histologia na totalidade dos esfregaços com atipias de significado indeterminado, LBG e LAG é uma estratégia de baixo custo e efetiva em situações de poucos recursos.

Com o presente trabalho, conclui-se que a prevalência de alterações tipo ASCUS, AGUS e lesões escamosas intra-epiteliais cervicais foi de $3,3 \%$, indice próximo do previsto pelo PNCC (4\%).

Nossos resultados demonstram que entre 57 pacientes, $27(47,3 \%)$ rastreadas citologicamente e incluídas no grupo das LBG apresentavam algum grau de NIC, chamando a atenção para $9(27,3 \%)$ NIC II e III, precursoras de alto grau que o esfregaço de Papanicolaou não detectou. Entretanto, o uso da colposcopia e da histologia fez com que se instituisse terapêutica mais adequada para esses casos. 
Das 16 pacientes com citologia oncológica compativel com lesões escamosas intra-epiteliais de alto grau, e que apresentaram anormalidades colposcópicas, $12,5 \%$ revelaram lesões de baixo grau ao estudo anatomopatológico, sendo, portanto, desnecessário o uso de CAF. No rastreamento de neoplasias cervicais, a colposcopia mostrou-se procedimento importante e indispensável.

\section{SUMMARY}

Purpose: to evaluate a populational sample of the screening proposed by the National Program of Uterine Cervical Cancer Control (PNCC), regarding the following issues: frequency of unsatisfactory cytologic results, cytologic frequency of atypical squamous or glandular cells of undetermined significance (ASCUS, AGCUS), low- or highgrade squamous intraepithelial lesions (SIL), comparing the cytologic results with anatomicopathological results of colposcopically directed biopsies.

Methods: through the written, broadcasting television and oral midia, women between 35 and 49 years were requested to have a preventive cytopathological test, to be collected by the authorized public health or other institutions accredited by SUS. The slides were analyzed by the programauthorized laboratories and all those patients from the populational sample from the municipality of Navirai in the State of Mato Grosso do Sul with cellular alterations were submitted to colposcopy and directed biopsy.

Results: the frequency of cytologic alterations of the ASCUS, AGCUS and SIL types was $3.3 \%$, an index that is close to that predicted by the PNCC (4\%); the percentage of samples that were unsatisfactory for evaluation was high (12.5\%); among the ASCUS, AGCUS or low grade-SIL patients, $27.3 \%$ presented intraepithelial lesions of a high grade in the anatomicopathological study; while patients with cytology compatible with high grade-SIL, the directed biopsy revealed that $12.5 \%$ presented low-grade intraepithelial lesions.

Conclusions: the choice of oncological cytology as the only method for the screening in the program allowed high indexes of false-negatives (27.3\%) and of false-positives (12.5\%). In the screening of cervical neoplasms, colposcopy has shown to be an important and indispensable method to guide the therapeutical management to be adopted.

KEY WORDS: Cervical cancer. Precancerous lesions. Cancer screening programs.

\section{Referências}

1. World Health Organization. WHO programme on cancer control. Developing a global strategy for cancer. Lyon: WHO; 1998. p.3.
2. Brasil. Ministério da Saúde. Secretaria Nacional de Assistência à Saúde. Instituto Nacional do Câncer. Estimativas da incidência e mortalidade por câncer no Brasil 2000. Rio de Janeiro: INCA; 2000.

3. Swinker M, Cutlip AC, Ogle D. A comparison of uterine cervical cytology and biopsy results: indications and outcomes for colposcopy. J Fam Pract1994; 38:40-4.

4. Miller AB, Nazeer S, Fonn S, et al. Report on consensus conference on cervical cancer screening and management. Int J Cancer 2000; 86:440-7.

5. Lachman MF, Cavallo-Calvanese C. Qualification of atypical squamous cells of undetermined significance in an independent laboratory: is it useful or significant? Am J Obst Gynecol 1998; 179:421-9.

6. Yang M, Zachariah S. ASCUS on cervical cytologic smears. Clinical significance. J Reprod Med 1997; 42:329-31.

7. Zweizig S, Noller K, Reale F, Collis S, Resseguie L. Neoplasia associated with atypical glandular cells of undetermined significance on cervical cytology. Gynecol Oncol 1997; 65:314-8.

8. Heatley MK, Bury JP. The correlation between the grade of dyskaryosis on cervical smear, grade of cervical intraepithelial neoplasia (CIN) on punch biopsy and the final histological diagnosis on cone biopsies of the cervix. Cytopathology 1998; 9:93-9.

9. Collaço LM, Tuon FFB, Soares MF, Totsugui JT, Torres LFB, Pinto AP. Correlação cito-histológica em 326 pacientes submetidas à cirurgia de alta freqüência $(\mathrm{CAF})$ no Programa de Prevenção do câncer ginecológico do Estado do Paraná. J Bras Patol 2000; 36:191-7.

10.Coppell K, Paul C, Cox B. An evaluation of the National Cervical Screening Programme Otago site. N Z Med J 2000; 113:48-51.

11.Bergström C, Sparén P, Adami HO. Trends in cancer of the cervix uteri in Sweden following cytological screening. Br J Cancer 1999; 81:159-66.

12.Flannelly G, Anderson D, Kitchener HC, et al. Management of women with mild and moderate cervical dyskaryosis. BMJ 1994; 308:1399-403.

13.Richart RM. Screening. The next century. Cancer 1995; 76 Suppl:1919-27.

14. Herbert A. Is cervical screening working? A cytopathologist's view from the United Kingdom. Hum Pathol 1997; 28:120-6.

15. Miller AB, Anderson G, Brisson J, et al. Report of a National Workshop on Screening for Cancer of the Cervix. CMAJ 1991; 145:1301-25.

16. Salmeron-Castro J, Lazcano Ponce EC, Perez Cuevas R, Del Rio Gomes I, Torres Torija I, Hernandez Avila M. Proposal to institutionalize criteria and quality standards for cervical cancer screening within a health care system. Cad Saúde Pública 1998; 14 Supl 3:67-75. 